\title{
Unraveling the role of secondary electrons upon their interaction with photoresist during EUV exposure
}

Ivan Pollentier, Yannick Vesters, Jing Jiang, Pieter Vanelderen, Danilo de Simone

Ivan Pollentier, Yannick Vesters, Jing Jiang, Pieter Vanelderen, Danilo de Simone, "Unraveling the role of secondary electrons upon their interaction with photoresist during EUV exposure," Proc. SPIE 10450, International Conference on Extreme Ultraviolet Lithography 2017, 104500H (16 October 2017); doi: 10.1117/12.2281449

SPIE Event: SPIE Photomask Technology and EUV Lithography, 2017, Monterey, California, United States 


\title{
Unraveling the role of secondary electrons upon their interaction with photoresist during EUV exposure
}

\author{
Ivan Pollentier*, Yannick Vesters, Jing Jiang, Pieter Vanelderen, and Danilo de Simone \\ Imec, Kapeldreef 75, B3001 Leuven (Belgium)
}

\begin{abstract}
The interaction of $91.6 \mathrm{eV}$ EUV photons with photoresist is very different to that of optical lithography at DUV wavelength. The latter is understood quite well and it is known that photons interact with the resist in a molecular way through the photoacid generator (PAG) of the chemically amplified resist (CAR). In EUV however, the high energy photons interact with the matter on atomic scale, resulting in the generation of secondary electrons. It is believed that these secondary electrons in their turn are responsible in chemical modification and lead to switching reactions that enable resist local dissolution. However, details of the interaction are still unclear, e.g. which reaction an electron with a given energy can initiate.

In this work we have introduced a method to measure the chemical interaction of the secondary electrons with the EUV resist. The method is based on electron gun exposures of low energy electrons (range $\sim 1 \mathrm{eV}$ to $\sim 80 \mathrm{eV}$ ) in the photoresist. The chemical interaction is then measured by Residual Gas Analysis (RGA), which can analyze out of the outgassing which and how much reaction products are generated. In this way a 'chemical yield' can be quantified as function of electron energy.

This method has been successfully applied to understand the interaction of secondary electrons on the traditional CAR materials. The understanding was facilitated by testing different compositions of an advanced EUV CAR, where resp. polymer only, polymer $+\mathrm{PAG}$, and polymer $+\mathrm{PAG}+$ quencher are tested with the electron gun. It was found that lowenergy electrons down to $\sim 3-4 \mathrm{eV}$ can activate PAG dissociation, which can lead to polymer deprotection. However it was observed too that energy electrons of $\sim 12 \mathrm{eV}$ and higher can do direct deprotection even in absence of the PAG. In addition, testing suggests that electrons can generate also other chemical changes on the polymer chain that could lead to cross-linking.
\end{abstract}

Keywords: EUV, photoresist, CAR, secondary electrons, RGA

\section{INTRODUCTION}

Optimizing the photoresist is one of the key challenges for high volume manufacturing (HVM) based on extreme ultraviolet (EUV) lithography. Compared to the lithography at DUV wavelength, the interaction of $91.6 \mathrm{eV}$ photons with photoresist is very different. In DUV lithography, most resist chemistries are based on chemically amplified resists (CAR), consisting of a polymer with protection groups (PG), blended with photoacid generator (PAG) and quencher (Q). Its photochemistry in DUV is well understood, and based on molecular interaction between photon and resist. In particular, the photon is absorbed by the PAG, which in turn can generate acids which is the starting point for the deprotection, which makes the exposed resist soluble for the developer after post-exposure bake. The role of the quencher is to balance the acid concentration at the edge of the exposed region and therefore improve line edge roughness (LER).

In EUV lithography, a lot of research is done into novel resist platforms, however still many CAR based chemistries are explored and optimized towards their use in HVM. In contrary to DUV, the interaction of $91.6 \mathrm{eV}$ photons with the resist is much less understood [1-2]. The reason for this is that these high energy photons not anymore react in a molecular way, but in a radiative way by generating photoelectrons by interactions with individual atoms in the resist. These electrons (which are expected to have energy around $80 \mathrm{eV}$ ) in their turn are believed to generate a cascade of secondary

* contact: ivan.pollentier@imec.be

International Conference on Extreme Ultraviolet Lithography 2017, edited by Paolo A. Gargini,

Patrick P. Naulleau, Kurt G. Ronse, Toshiro Itani, Proc. of SPIE Vol. 10450, 104500H

(C) 2017 SPIE $\cdot$ CCC code: $0277-786 X / 17 / \$ 18 \cdot$ doi: $10.1117 / 12.2281449$ 
electrons with lower energy, which eventually should result in a chemical reaction where acid is created by the PAG. However, there are many aspects not fully understood :

- Which atoms generate the electrons of interest?

- How much secondary electrons can a photon generate ?

- What energy distribution do the secondary electrons have ?

- Which chemical reaction can secondary electrons cause, and what is the minimum energy to do that ?

In this work, the focus is to provide more understanding on the last question, and clarify how electrons with varying energy can introduce chemical reactions in CAR based material. Given the complexity described above, the understanding is enabled by introducing simplifications.

A first simplification that is introduced is that electrons (instead of photons) are used as exposure source on the resist, by using an electron gun. By proper choice the e-gun, electrons of one selected energy only can be exposed on the resist and this in the energy range that is relevant for the EUV resist (i.e. $\sim 1 \mathrm{eV}$ to $\sim 80 \mathrm{eV}$ ).

A second item in the complexity is the resist itself. The CAR is a mixture of polymer, PAG, and quencher, so the exposed electrons have potentially different materials to interact with. So the other simplification is to do the testing not only on the full CAR composition, but also do parallel testing on compositions where e.g. quencher and/or PAG is not included. In this way it was possible to differentiate the electron-induced reaction with e.g. the polymer to that of the PAG.

\section{EXPERIMENTAL AND MEASUREMENT PROCEDURE}

In this work, CAR based materials are investigated with low energy electrons. The main CAR material in this work was based on good NXE-performing (commercial) resist that was provided in 3 versions :

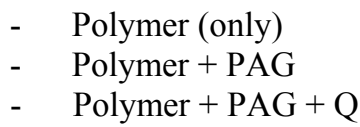

Exposures and measurements in this work are done on EUV Tech outgas tool in imec, used earlier for resist outgas qualification [3]. This tool enables to expose a $200 \mathrm{~mm}$ resist coated wafer either by EUV photons either by electrons. For the EUV exposure, an Energetiq EQ-10 is integrated in the tool. For electron based exposures a low-energy electron gun ELG-2 from Kimball Physics has been installed, which can expose electrons with energy from $1 \mathrm{eV}$ to $2 \mathrm{keV}$. For the results reported in this paper, the range $1-80 \mathrm{eV}$ was mostly used. Faraday cup measurements showed that the electron current is fairly constant when changing the energy in this range.

In analogy with other reports [4], the measurement of the chemical modification in the photoresist is done by Residual Gas Analysis (RGA). In the outgas tool this was done with a Pfeiffer QMG422 mass spectrometer, which can measure over a broad atomic mass (amu) range of 1 to 512amu. So, the over-all principle used here to measure electron-induced chemical modification is to do simultaneous electron exposure and RGA measurement. This is schematically illustrated in Fig. 1, and details of the procedure are described below.

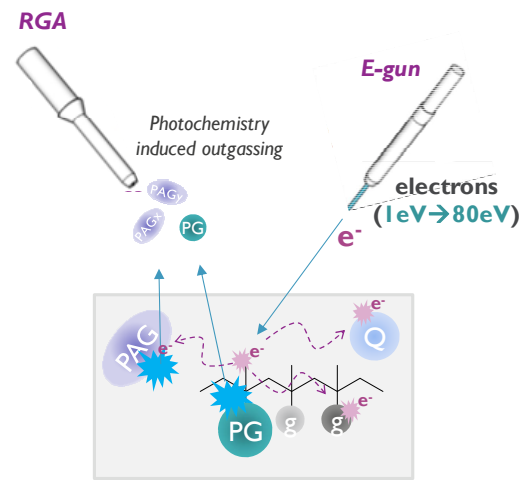

Figure 1. Schematic representation of the principle of measurement of electron induced chemical reaction. 


\subsection{Determination of species and relevant masses}

For many photoresists the range 1-300amu is sufficient to detect all the resist related outgassing, However the full spectrum measurement is time-consuming, and difficult to use in combination with electron gun exposures. Therefore, upfront of the electron testing, full spectrum measurement were done on the resists to identify the main masses of the outgas species of the three resists indicated above. To be closest to the EUV application, the Energetiq exposure was here (although earlier work [2] gave evidence that the outgas spectrum by EUV and electrons is not significantly different). Having the resist available in different compositions, was significantly simplifying the interpretation of the spectra : in the RGA test done on the polymer, only polymer related reaction products are seen (mostly protection groups), while in the test with polymer+PAG, the polymer spectrum could be subtracted, resulting in only PAG related species. In this way, the relevant masses (amu) could be obtained for the different chemical reactions involved, and it could be traced back which mass is relevant for the different resist components.

\subsection{RGA measurement during electron exposure}

Once the masses of the species are identified that are relevant for the chemical reaction, it is possible to do fast RGA acquisition on only these masses. This approach, where each few seconds a time-registration is done on the presence on one of the outgas species, is now applied in combination electron gun exposures, and illustrated in Fig. 2. In this figure, the methodology is explained by only one of the above mentioned resist compositions, viz.. polymer+PAG, where PAG and PG reactions and species are expected.

The electron exposure on this photoresist coated wafer is done in a line scan (Fig. 2 (b)), where from line to line the electron energy is increased. A reference scan without electron exposure (' $0 \mathrm{eV}^{\prime}$ ') is used as reference for RGA, while in between the lines the exposure is also interrupted. The measured RGA signal for two masses (one for PG and one for PAG) during this exposure is shown in Fig. 2 (a). It can be seen that the RGA detects already a significant signal for the $\mathrm{PAG}$ at $2 \mathrm{eV}$, whereas the PG shows that only around $4 \mathrm{eV}$. It can also be observed that every time the electron exposure is stopped in between the lines, the signal drops significantly, but that this local minimum is increasing in time. The reason for that increase is residual outgassing : when a line at e.g. $30 \mathrm{eV}$ is exposed, not only outgassing is detected from that exposure, but also the ongoing chemistry in earlier exposed lines will contribute to the RGA measurement.

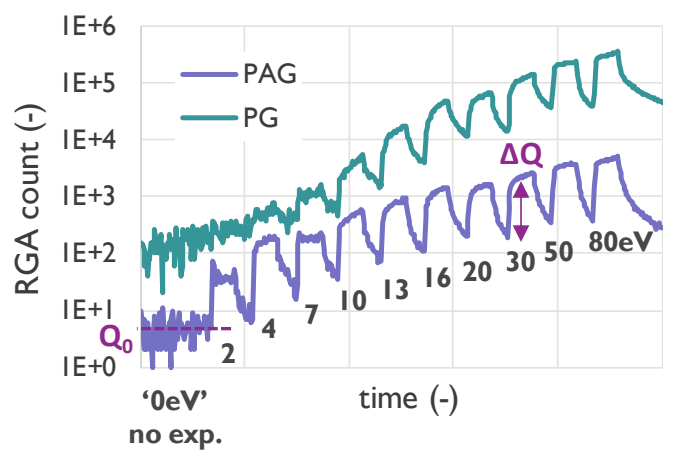

(a)

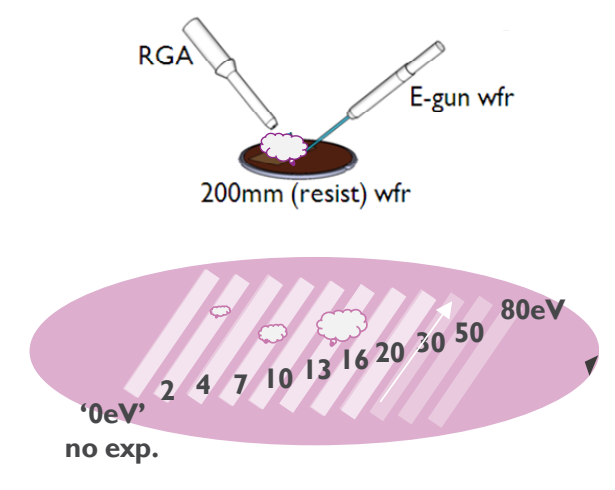

(b)

Figure 2. Details of the measurement : (a) time registration of RGA signal at masses related to PAG and PG; this is measured during line-scan exposure by the e-gun, shown in (b); each line is exposed at constant electron current, but electron energy is changed as indicated from line to line.

\subsection{Chemical yield analysis}

Although the time registration of RGA in Fig. 2 (a) is already of qualitative interest to identify at which energy the chemical reactions are observed, the aim was to get a more quantitative metric out of the signal in order to compare the chemical impact of the different electron energies. Since the over-all RGA signal is a superposition of residual outgassing, it was considered that the incremental increase $\Delta Q$ at the start of a line exposure is a good measure for the 
impact that this energy has on chemical reactions. Moreover, since the magnitude of RGA count is depending on many parameters, it is believed that a normalization to its initial value $\mathrm{Q}_{0}$ (during reference scan without exposure) is important to include also. Since the metric is showing a result of chemical reaction it is called chemical yield and given by $\Delta \mathrm{Q} / \mathrm{Q}_{0}$.

For the example RGA scan given in Fig. 2, the dependency of the chemical yield as function of the electron energy is shown in Fig. 3 (a). Both for the PAG and PG, the chemical yield is very low at energies lower than 10eV. From then on a clear increase is observed for both species, where that for PG is larger and for PAG.

As indicated earlier, the electron current for each energy is fairly constant in this measurement result, only the electron energy is changed. So one check to evaluate the consistency of the chemical yield measurement, would be to look to chemical yield results at different currents. This is illustrated in Fig. 3 (b) and (c) where similar tests were done at resp. much lower electron current and much higher electron current. It can be seen that the over-all trend at low current is very similar to that of Fig. 3 (a), the main difference is that the increase as function of electron energy is less steep and the yield at highest energy is much smaller. For the case of Fig. 3 (c) where the test was done at much higher current, the shape of the curve was slightly different. In particular, at energies higher than $20 \mathrm{eV}$ a flattening is observed, and the over-all magnitude of the yield is still comparable to that of Fig. 3 (a). It is believed that this different behavior at high current is due to the lack of available chemicals compared to the available electrons, so the testing at very high current seems to be in an chemical-limited regime. For the cases of Fig. 3 (b) and (a), there is not yet a sign of depletion of chemicals, so it is expected that the testing there is in the electron-limited regime. Although it is difficult to evaluate which case of Fig. 3 is closest to typical EUV exposures, it is expected that here the number of electrons will be small compared to the available chemicals, so in the remainder of the work electron-limited conditions.

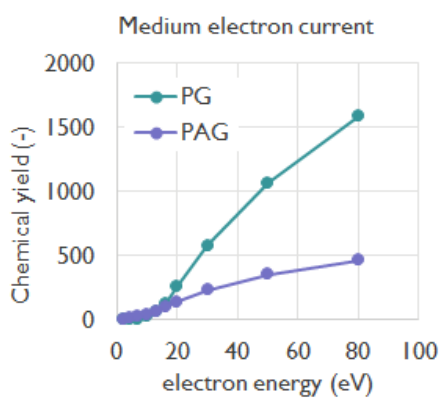

(a)

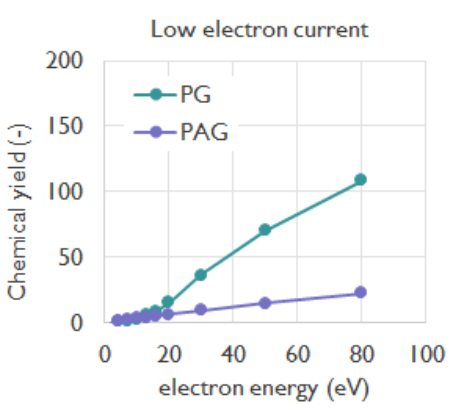

(b)

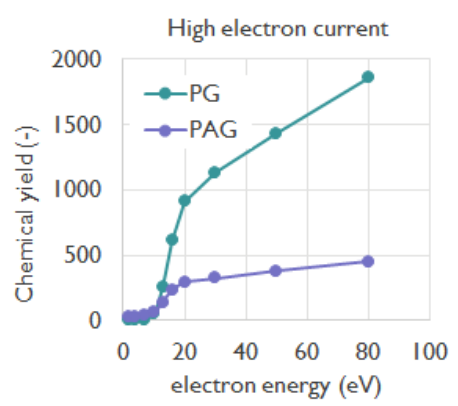

(c)

Figure 3. Chemical yield measurement results for the polymer+PAG resist, tested at three electron currents (see text).

\section{ELECTRON-INDUCED CHEMICAL INTERACTION IN CAR}

In this part the results of chemical yield measurements exposed at different electron energies will be discussed for CAR based materials. The main CAR material in this work was based on good NXE-performing (commercial) resist that was provided in 3 versions :

- Polymer (only)

- $\quad$ Polymer + PAG

- $\quad$ Polymer $+\mathrm{PAG}+\mathrm{Q}$

The main goal of the investigation was to have better understanding on which chemical reactions the electrons can do in the CAR and at which energy they can start chemical changes.

\subsection{PAG acid activation}

Equivalent to DUV lithography, where all photons are expected to do only PAG chemical interactions, and hence leading to acids only, the ideal scenario in EUV for the CAR is that all the secondary electrons generated by the photons are used for PAG reactions only, and that even low energy electrons can do the PAG chemical reaction.

In Fig. 3 above, it was already indicated that electron energy below $10 \mathrm{eV}$ starts to do PAG activation, and even the raw RGA scan of Fig. 2 was suggesting that PAG reaction is already observed at $2 \mathrm{eV}$. In order to clarify the chemical impact 
of low energy electrons to the PAG, the chemical yield was investigated in more detail in the range $1 \mathrm{eV}$ to $10 \mathrm{eV}$. The result of the measurement is shown in Fig. 4. It was found that even at electron energy as low as $1 \mathrm{eV}$ show a significant RGA signal for PAG, resulting in a chemical yield of about 10. However, as can be seen, this chemical yield is not significantly increasing towards a higher electron energy up to $\sim 3 \mathrm{eV}$. When electron energy is larger than $3 \mathrm{eV}$, the chemical yield starts to be proportional to the electron energy. It is believed that this onset - in between $3 \mathrm{eV}$ and $4 \mathrm{eV}-$ should be considered as real minimum electron energy for PAG and acid activation. The clear RGA signal at lower electron energy is not fully understood. One reason could be related to the fact that the electrons are generated outside the photoresist and need to pass the resist surface, where PAG molecules might have different way of activation.

More evidence that $3 \mathrm{eV}$ to $4 \mathrm{eV}$ is more relevant for PAG activation energy is found in the analysis of the RGA signal for the PG, which is also present in Fig. 4. For energies below 4eV, the RGA signal for the PG is at noise level, only from $4 \mathrm{eV}$ onwards, a significant chemical yield was observed. As will become clear in next section, this deprotection is solely due to the activated PAG, and in indirect way this confirms that below $3-4 \mathrm{eV}$ no PAG related acid is available for deprotection.

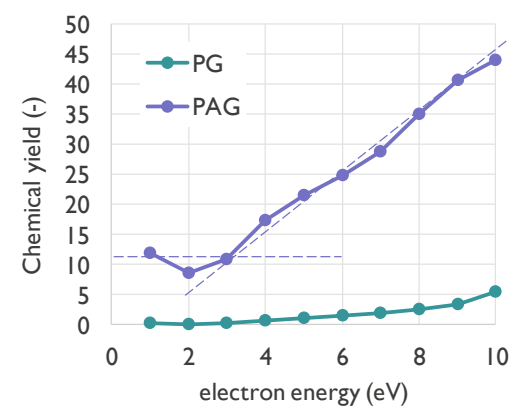

Figure 4. Chemical yield results for the polymer+PAG resist, measured in electron energy range of $1 \mathrm{eV}$ to $10 \mathrm{eV}$. The activation energy to generate PAG acid should be interpreted as the onset where chemical yield is proportional to electron energy which is in between $3 \mathrm{eV}$ and $4 \mathrm{eV}$.

\subsection{PG deprotection}

The chemical yield analysis discussed above for the polymer+PAG resist composition suggest that the observed PG deprotection can be a result of the acid interaction after the PAG dissociation. It is however reported in literature that EUV exposure can also direct scission of the PG [5]. Here this work, we made a first order assessment on the relative contribution of the direct scission to that of the PAG acid, by comparing the PG chemical yield of the polymer+PAG resist to that of the polymer-only.

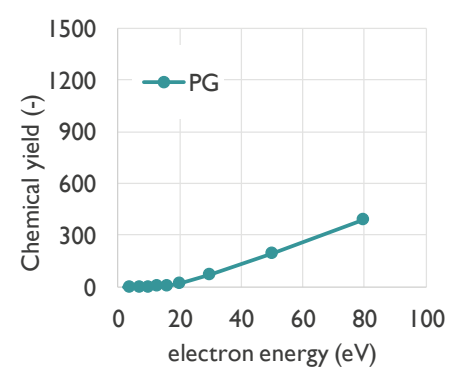

(a)

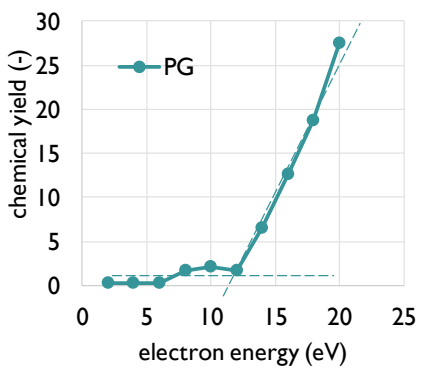

(b)

Figure 5. Chemical yield results for the polymer-only resist; in (a) testing was done in same energy range and current as the polymer+PAG test from Fig. 3(a). This comparison indicates that $\sim 15 \%$ of PG yield seen for the polymer+PAG is due to direct scission; In (b) it is shown that the activation energy for direct scission is about $12 \mathrm{eV}$. 
This results of this comparison are shown in Fig. 3 (a) and Fig. 5 (a), where the chemical yield of resp. polymer+PAG and polymer-only resist are shown for a same range of electron energies and at same current. In case of the polymer+PAG, the PG chemical yield is clearly higher than that of polymer-only, which indicates that the majority of deprotection observed is due to PAG acid formation. However, the PG chemical yield for the polymer-only is still in the order of $15 \%$, which could suggest that a signifcant number of secondary electrons are used for the scission and therefore not available anymore for acid formation, if e.g. electron attachment [2] is involved in that.

In Fig. 5 (b), the electron-induced chemical impact is investigated at a smaller energy range in between $2 \mathrm{eV}$ and $20 \mathrm{eV}$, in order to evaluate the minimum electron energy that is required for direct scission. Similar as for the PAG in Fig. 4, a small signal is observed already below $10 \mathrm{eV}$, however the onset for proportional increase with electron energy is from $12 \mathrm{eV}$ onwards. This energy is believed as real electron energy for the PG scission.

\subsection{Other chemical reactions?}

Besides the PAG and PG related outgassing, the investigated resist also showed $\mathrm{CO}_{2}$ as outgas product. By breakdown of the analysis into the different resist compositions, it was found that $\mathrm{CO}_{2}$ was related to the polymer. Although details of the polymer structure of this resist was unknown, it is reported in literature that $\mathrm{CO}_{2}$ is a possible reaction product in EUV for acrylate related photoresist as a result of Norrish type1 reaction and ester elimination [5]. This chemistry is frequently used in EUV resist, which could explain why the $\mathrm{CO}_{2}$ is observed here but also for many other CAR materials in earlier outgas testing with EUV exposure.

In Fig. 6 (a) the chemical yield analysis was investigated for its interaction with electrons, which shows that its activation energy for the $\mathrm{CO}_{2}$ reaction is about $20 \mathrm{eV}$. Although the yield levels seem small, the testing gives evidence that a number of secondary electrons are used for this reaction. Besides that fact that the electrons are not anymore available for acid formation, it is expected that the Norrish type 1 reaction of $\mathrm{CO}_{2}$ will result in a carbon radical on the polymer matrix, which can result in polymer cross-linking.

In order to check if the cross-linking suggested by the $\mathrm{CO}_{2}$ outgassing has impact for this resist in EUV printing conditions, a contrast curve test of the polymer $+\mathrm{PAG}+\mathrm{Q}$ resist was done using EUV exposure, of which the result is given in Fig. 6 (b). In this test the resist is exposed with increasing dose, and after PEB and development, the resist thickness is measured in the flood exposed areas. As can be seen, the resist had a dose-to-clear of about $20 \mathrm{~mJ} / \mathrm{cm}^{2}$, which was in expected relationship with the dose-to-size of the resist for printing critical dimensions on the EUV scanner. In the contrast curve, it can be seen that at high doses the resist is not developable anymore, which gives evidence of the cross-linking by EUV exposure. However, the exposure dose required is about $200 \mathrm{~mJ} / \mathrm{cm}^{2}$, which is much higher than the doses used for printing in EUV scanner. Nevertheless, further investigation could be important to exclude that risk more in detail.

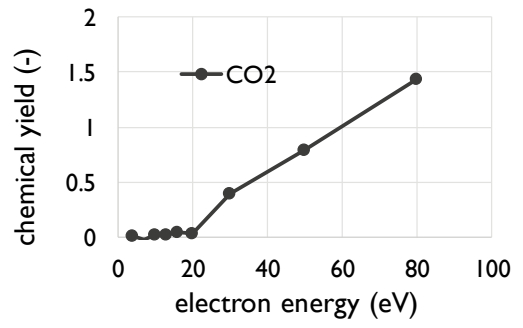

(a)

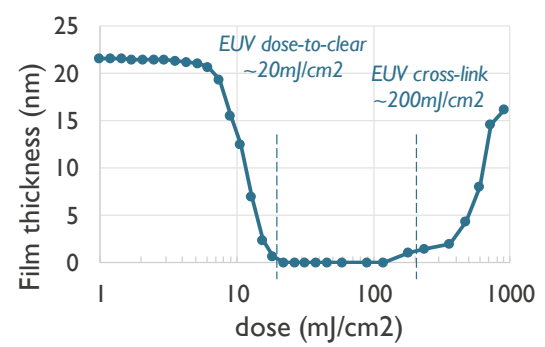

(b)

Figure 6. (a) Chemical yield testing as function of electron energy shows an activation energy of $\sim 20 \mathrm{eV}$ for the $\mathrm{CO}_{2}$ scission; (b) since literature suggest a cross-linking of the polymer related to this scission, a contrast curve test was done to check the impact in EUV exposure conditions. It was found that the cross-linking occurs at $\sim 10$ times higher dose than the dose typically used for scanner applications. 


\section{CONCLUSIONS}

In this paper, we introduced a methodology to measure the chemical impact of secondary electrons in EUV resists. The method is based on low energy electron-gun exposures, while simultaneous Residual Gas Analysis measures the response of outgassed photochemical reactions. The electron energy can be tuned by the electron gun, which enables to quantify the chemical yield of a reaction as function of electron energies.

This methodology has been applied to an EUV photoresist supplied in different compositions : polymer-only, polymer $+\mathrm{PAG}$, and polymer $+\mathrm{PAG}+\mathrm{Q}$. This enabled the possibility to determine which electron-induced reactions can occur with the individual chemicals of the photoresist. It has been found that PAG and acid activation starts at electron energy of $3-4 \mathrm{eV}$. But not all electrons are used for PAG/acid activation. A limited amount $(\sim 15 \%$ is estimated for this resist) seems to be used for direct scission of the protection group from the polymer chain. Electrons with energy $\sim 12 \mathrm{eV}$ are required for this. In addition, there is evidence that $\mathrm{CO}_{2}$ is also leaving from polymer chain. Electrons with energy of $\sim 20 \mathrm{eV}$ are required for this. There is evidence that polymer cross-linking can be induced by that at high dose EUV exposure, but it is not clear if this can influence the scanner printing performance, which is done at much lower dose-tosize.

\section{ACKNOWLEDGEMENTS}

The authors like to thank Geert Vandenberghe (imec) and John Petersen (Petersen Advanced Lithography and imec) for helpful discussions on CAR chemistry and measurements. In addition, we acknowledge Alex Vaglio Pret (KLA-T) and Peter De Schepper (Inpria) for discussions on electron exposures, and the resist suppliers for providing photoresist in different compositions. Finally, we would like to thank Dave Houser and Derek Yegian (EUV Tech) and Tyler Ruttan (Kimball Physics) for helpful discussions on the low energy electron-gun.

\section{REFERENCES}

[1] Kozawa, T. and Tagawa, S, "Radiation chemistry in chemically amplified resists," Jpn. J. Appl. Phys. 49, $030001(2010)$.

[2] Van Dorp, W.F., [Materials and Processes for Next Generation Lithography - Theory : electron-induced chemistry], London: Elsevier Ltd., 115-133 (2016).

[3] Pollentier, I., Lokasani, R., Gronheid, R., Hill, S., Tarrio, C., and Lucatorto, T., "Relationship between resist related outgassing and witness sample contamination in the NXE outgas qualification using electrons and photons," Proc. SPIE 8679, 86790K-1-12 (2013).

[4] Grzeskowiak, S., Narasimhan, A., Ostrander, J., Schad, J. Earley, W., Brainard, R.L., and Denbeaux, G., "Cross-sections of photoacid generators at low electron energies," J. Vac. Sci. Technol. B (33(6), (2015).

[5] Fedynyshyn, T.H., Goodman, R.B., Cabral, A., Tarrio, C., and Lucatorto, T.B., "Polymer photochemistry at the EUV wavelength," Proc. SPIE 7639, 76390A-1 (2010). 